\title{
Autoestima y consumo de alcohol en adolescentes de secundaria de Ciudad del Carmen, Campeche
}

\author{
Telumbre-Terrero, Juan Yovani1*; Lopez-Cisneros, Manuel Antonio²; Noh-Moo, Pedro Moisés3; \\ Villanueva Echavarria, José Rafael4; Torres-Obregón, Reyna ${ }^{5}$
}

\begin{abstract}
RESUMEN
Introducción: Los adolescentes transitan por una etapa de cambios biológicos, psicológicos y sociales que pudieran ser factores condicionantes en la adquisición de conductas nocivas como el uso y/o abuso de alcohol. Objetivo: Establecer la asociación entre autoestima y el consumo de alcohol de los adolescentes. Metodología: Estudio descriptivo correlacional llevado a cabo en 191 adolescentes de Educación Secundaria de Ciudad del Carmen Campeche, México. Se utilizó la Prueba de Identificación de Desórdenes por Uso de Alcohol (AUDIT) y la Escala de Autoestima de Rosenberg. Resultados: Predomina el tipo de consumo de dependiente (35.4\%) seguido del sensato (34.1\%), por sexo en los varones predomina un consumo dependiente (39.4\%) y en las mujeres un consumo sensato (38.8\%). Se estableció una relación negativa entre el autoestima y consumo de alcohol de acuerdo a la puntuación AUDIT $(r=-.425, p=.042)$. Conclusión: La ingesta de bebidas alcohólicas representa un problema creciente de salud que pudiera ocasionar múltiples daños a la salud de los adolescentes, además de estar asociado a variables psicológicas como la autoestima, que juega un papel primordial en la adquisición o modificación de hábitos saludables o no, y por lo tanto constituye un factor protector ante el consumo de alcohol.
\end{abstract}

Palabras claves: Adolescente, Consumo de Bebidas Alcohólicas, Autoestima (DeCS;BIREME).

${ }^{1}$ Maestro en Ciencias de Enfermería. Universidad Autónoma del Carmen- Facultad de Ciencias de la Salud- Licenciatura en Enfermería- Ciudad del Carmen Campeche, México. E-mail: jtelumbre@pampano.unacar.mx

${ }^{2}$ Doctor en Ciencias de Enfermería. Universidad Autónoma del Carmen- Facultad de Ciencias de la Salud- Licenciatura en Enfermería- Ciudad del Carmen Campeche, México.

${ }^{3}$ Licenciado en Enfermería. Universidad Autónoma del Carmen- Facultad de Ciencias de la Salud- Licenciatura en Enfermería- Ciudad del Carmen Campeche, México.

${ }^{4}$ Maestro en Ciencias de la Salud. Universidad Autónoma del Carmen- Facultad de Ciencias de la Salud- Licenciatura en Enfermería- Ciudad del Carmen Campeche, México.

${ }^{5}$ Doctora en Ciencias de Enfermería. Universidad Autónoma de Coahuila. Facultad de Enfermería- Saltillo Coahuila, México

*Autor para correspondencia

Cómo citar este artículo

Telumbre-Terrero JY, Lopez-Cisneros MA, Noh-Moo PM, Villanueva-Echavarria JR, Torres-Obregón R Autoestima y consumo de alcohol en adolescentes de secundaria de Ciudad del Carmen, Campeche. Sanus. 2018;3(6): 20-31. [Acceso ]; Disponible en: mes día año 


\section{INTRODUCCIÓN}

El alcohol es una sustancia ampliamente utilizada y que ocasiona efectos negativos en las personas que lo consumen en exceso, además de problemas a nivel familiar, laboral y social, lo cual implica costos directos en los diversos sectores de la sociedad. Estos efectos estarán determinados por tres aspectos fundamentales: volumen de alcohol consumido, frecuencia y cantidad consumida y en raras ocasiones por la calidad del alcohol(1).

Anualmente, en el mundo se originan más de 3 millones de muertes ocasionadas por el consumo de alcohol, además constituye un factor etiológico de múltiples problemas de índole biológico psicológico y social, tales como: cirrosis hepática, cáncer, enfermedades cardiovasculares, suicidio, depresión, violencia y accidentes de tránsito entre otros (2).

En México se ha identificado que 25 de cada 100 adolescentes manifestaron haber ingerido alguna bebida alcohólica durante el último año, 11 de cada 100 adolescentes varones consumieron más de cinco bebidas estándar en una sola ocasión de consumo; el 1\% de los adolescentes abusa diariamente, $2.3 \%$ semanal, $5.8 \%$ mensual y $7.2 \%$ de manera ocasional ${ }^{(3)}$. Aunado a lo anterior se han identificado que son diversas causas que condicionan el comportamiento de los adolescentes. Esta vulnerabilidad resulta de los cambios característicos de esta etapa a nivel neurológico, cognitivo y social. Esta última supone que los adolescentes construyan su identidad personal ante la familia, escuela y amigos, considerando también la adquisición de comportamientos saludables o no saludables, dentro de los cuales se ubica el consumo de drogas ${ }^{(4)}$.

Un factor protector y condicionante ante el no consumo de drogas es la autoestima, la cual es definida por Rosenberg ${ }^{(5)}$ como el valor que cada persona tiene de sí mismo, retomando experiencias, sensaciones y emociones vividas durante las etapas de la vida. Esta valoración que el sujeto hace de sí mismo es imprescindible para el proceso adaptativo con los demás y la sociedad ${ }^{(6)}$. Hay que considerar que la adolescencia representa una etapa crucial donde los adolescentes presentan mayores exigencias sociales, como por ejemplo la definición de la identidad, autonomía, mayor interacción con otras personas y del sexo opuesto, entre otras que pueden afectar el nivel de la autoestima. Por lo tanto la autoestima pudiera determinar la reacción ante los acontecimientos de la vida y la capacidad de afrontamiento, coadyuvando a la mejor toma de decisiones ante los problemas que el sujeto pudiera tener en esta etapa de vulnerabilidad(7).

La autoestima proporciona al adolescente las capacidades necesarias para que pueda resistir a la presión de grupo ante el consumo de drogas; y así poder tomar decisiones saludable ${ }^{(8)}$. La autoestima es una variable psicológica reguladora durante la adquisición de conductas y comportamientos saludables o no saludables de los adolescentes, y dependerá del grado de autoestima que posean los adolescentes y jóvenes en un determinado momento para la adopción o modificación de los estilos de vida ${ }^{(9)}$.

Por lo anteriormente expuesto el objetivo de la presente investigación fue establecer la relación de la autoestima y el consumo de alcohol en adolescentes, esto permitirá en un primer momento profundizar en el conocimiento de la autoestima como variable mediadora de conductas saludables o no saludables de los adolescentes. En un segundo plano servir de sustento teórico para los profesionales de la salud, específicamente para enfermería en relación a la promoción y educación para la salud, por medio de acciones específicas en el fortalecimiento de los factores de protección. Además de identificación y canalización oportuna de los adolescentes que requieran apoyo para disminuir o suprimir el consumo de esta u otras sustancias.

\section{METODOLOGÍA}

Investigación de tipo descriptivo correlacional(10) llevado a cabo en noviembre de 2017 en una institución pública de Educación Secundaria de Ciudad del Carmen Campeche, México con una matrícula general de 658 alumnos de ambos sexos. Se realizó un muestreo aleatorio estratificado por semestre(11) y la muestra se calculó en el software estadístico n'Query Advisor Versión 4.0 (12), bajo los siguientes parámetros: potencia 90\%, significancia .05, coeficiente de correlación alterno .18; quedando una muestra final de 191 participantes.

Estudio aprobado por el Comité de Ética e Investigación del Departamento de Investigación y Posgrado de la Universidad Autónoma del Carmen con el registro FCS/2016/07 y así como de la institución en la cual se realizó el estudio. Posteriormente se solicitó la lista de alumnos legalmente inscritos en el ciclo escolar 2017, los participantes fueron seleccionados al azar. Una vez seleccionados los participantes se hizo entrega del consentimiento informado para tutores y el asentimiento informado para los menores de edad. Obtenida la autorización por ambas partes se hizo entrega del instrumento de medición, y dando lectura a las instrucciones de llenado. En todo momento se respetó la integridad de los participantes y así como lo estipulado en el Reglamento de la Ley General de Salud en Materia de Investigación para la Salud ${ }^{(13)}$. Al finalizar el llenado de los cuestionarios, estos fueron colocados en una urna ubicada en la entrada del aula asignada por la institución.

Para la recogida de la información se utilizó una ficha datos personales y antecedentes del consumo de alcohol, que indaga sobre datos de identificación, sociodemográficas y características de la ingesta de alcohol. También se utilizó la Prueba de Identificación de Desórdenes por Uso de Alcohol (AUDIT), este instrumento consta de 10 preguntas de opción múltiple que permite identificar a los individuos con problemas del uso y abuso del alcohol(14). Las puntuaciones de la escala fluctúan entre 
0 a 40, lo cual indica que a mayor puntaje mayor es el riesgo de problemas de dependencia al alcohol. Para una mejor interpretación puntuaciones entres 1-3 indican un consumo sensato, de 4-7 consumo dependiente y de 8- 40 puntos se considera consumo dañino. Esta escala ha sido adaptada y validada en población mexicana reportan una sensibilidad y especificidad aceptable(15), en otros estudios ${ }^{(16,17)}$ se reporta una confiabilidad de $.74-.87$, en este estudio obtuvo una confiabilidad de $\alpha=0.77$.

La Escala de Autoestima de Rosenberg, constituida por 10 afirmaciones, con una escala de respuesta de 1 a 4 puntos, para su interpretación puntuaciones entre 30 a 40 puntos indican autoestima alta, de 26 a 29 autoestima normal y puntuaciones de 25 o menos indican autoestima baja ${ }^{(18)}$. Esta escala ha reportado una confiabilidad aceptable en población adolescentes de Querétaro(19) y Nuevo León ${ }^{(7)}$, para esta investigación se obtuvo una confiabilidad de $\alpha=$ 0.89 .

En análisis e interpretación de la información se realizó en el programa estadístico Statistical Package for the Social Sciences (SPSS), versión 23.0 para Windows, mediante el uso de estadística descriptiva (frecuencias, proporciones, medidas de tendencia central y de dispersión) e inferencia paramétrica dado los resultados de la prueba de normalidad de para las variables continuas.

\section{RESULTADOS}

El $50.8 \%$ de los adolescentes pertenecen al sexo masculino, con una media de edad de 13.4 años $(D E=2.1)$, por lo que respecta al grado escolar el 44\% está ubicado en el primer año y el $41.9 \%$ en tercer año, además el $78 \%$ de los adolescentes viven con ambos padres, $12.6 \%$ solo con mamá y el $2.6 \%$ solo con papá. Al indagar sobre la ingesta de bebidas alcohólicas de algún integrante de la familia, el 60.7\% respondió afirmativamente, siendo el padre el principal consumidor (24.6\%), seguido de ambos padres (13.1\%) y hermanos (10.5\%), consumiendo hasta 17.1 (DE= 8.3) bebidas en un día típico de consumo. Por lo que se refiere a los adolescentes se identificó que el $65.4 \%$ han ingerido alguna bebida alcohólica en algún momento de su vida y el $8.4 \%$ en la última semana (Tabla 1).
En cuanto a los reactivos que evalúan las características de la autoestima, se pude destacar que el $56.6 \%$ de los adolescentes están totalmente de acuerdo que son un fracaso y que no sirven para nada con un 54.6\%, además que algunas veces se sienten inútil (42.9\%) y que no tienen algo por que sentir orgullo (32.5\%). Por otro lado, el 51.3\% están totalmente de acuerdo que es una persona de valor, tener una actitud positiva y estar satisfecho con su persona (Tabla 2).

Se identificó que en el $80.8 \%$ de los adolescentes predomina el nivel de autoestima baja, siendo mayor mujeres (81.4\%) en relación con los varones (79.8\%). Respecto a los puntos de cohorte del AUDIT, predomina el tipo de consumo de dependiente (35.4\%) seguido del sensato (34.1\%), por sexo en los varones predomina un consumo dependiente (39.4\%) y en las mujeres un consumo sensato (38.8\%) (Tabla $3)$.

Por lo que se refiere a la prevalencia global del consumo de alcohol por nivel de autoestima, se pudo identificar que la autoestima baja (33.8\%) y autoestima media (7.9\%) predomina en aquellos adolescentes que han ingerido alcohol en algún momento de su vida (Tabla 4).

En relación al objetivo de la investigación se determinó una relación negativa y significativa entre en nivel de autoestima y el consumo de alcohol de acuerdo a la puntuación AUDIT ( $r=-.425, p=.042$ ) y numero de bebidas alcohólicas consumidas en un día típico ( $r=-.367, p=.039$ ).

\section{DISCUSIÓN}

Los hallazgos evidencian que una cuarta parte la población manifestó haber consumido alcohol en el último mes, lo cual concuerda con investigaciones realizadas en Estados del Sur ${ }^{(20,21,22)}$ de México. Cabe destacar las características de la ingesta (cantidad y frecuencia) de alcohol, son dos aspectos fundamentales que se deben de evaluar y que son cruciales para identificar dependencia alcohólica ${ }^{(23,24)}$. Además de considerar la interacción de factores de índole personal, psicológico, familiar y social como condicionantes en la adquisición de la conducta del consumo de alcohol(25, 26).

Se identificó una autoestima baja, siendo mayor en las mujeres que en los varones, esto es similar a lo reportado

Tabla 1. Prevalencias de consumo de alcohol

\begin{tabular}{lccccc}
\hline & Prevalencia & \multicolumn{2}{c|}{ Si } & \multicolumn{2}{c}{ No } \\
\cline { 2 - 5 } & Global & $f$ & $\%$ & $f$ & $\%$ \\
\cline { 2 - 5 } Lápsica & 125 & 65.4 & 66 & 34.6 \\
Actual & 82 & 42.9 & 109 & 57.1 \\
Instantánea & 49 & 25.7 & 142 & 74.3 \\
\hline Fuente: Elaboración propia & 16 & 8.4 & 175 & 91.6 \\
\hline
\end{tabular}


por un grupo de investigadores de España ${ }^{(6)}$. Esto pudiera estar relacionado a dos aspectos cruciales; primero, las cuestiones laborales donde la incorporación de la mujer en este medio ha traído consigo una igualdad en las condiciones laborales, y segundo, las cuestiones de género, donde existe poca aceptación de los varones ante la incorporación de las mujeres en actividades socialmente activas, lo cual pudiera explicar la disminución de la autoestima femenina $(27,28)$. Cabe destacar también que durante la adolescencia las cuestiones biológicas juegan un papel fundamental ya que son mucho más marcadas en las adolescentes y por supuesto las cuestiones culturales donde se construye la identidad como hombre o mujer ${ }^{(29,30)}$.

Además prevaleció el consumo dependiente y sensato de manera global, en los varones un consumo dependiente y en las mujeres un consumo sensato, lo cual difiere con los hallazgos reportados en adolescentes de secundaria de Querétaro,México(19), donde predomina un consumo sensato lo cual pudiera estar vinculado a la incorporación de mujeres en actividades remuneradas con acceso a puestos gerenciales y mayor remunerados, así como acceso a los servicios educativos permitiéndoles formación académica con niveles de licenciatura, maestría y doctorado(31).

En consonancia con el objetivo de esta investigación se identificó una relación negativa y significativa de la autoestima y el consumo de alcohol, lo cual indica que a mayor autoestima menor consumo, concordando con investigaciones nacionales ${ }^{(7,9)}$, que indican que la autoestima representa un factor de protección ante el consumo de drogas lícitas e ilícitas. Es así que la baja autoestima conlleva a una inadecuada adaptación de los adolescentes con su medio familiar y social, lo cual le impide superar los desafíos de la vida diaria de la mejor manera. Por el contrario, adolescentes con autoestima alta son más seguros de sí mismo, presentan alta confianza y por ende son competentes con la vida personal y profesional ${ }^{(32)}$.

Tabla 2. Frecuencias de los reactivos de la Escala de Autoestima de Rosenberg

\begin{tabular}{|c|c|c|c|c|}
\hline \multirow[t]{2}{*}{ Ítem } & $\begin{array}{l}\text { Totalmente en des- } \\
\text { acuerdo }\end{array}$ & En desacuerdo & De acuerdo & $\begin{array}{l}\text { Totalmente } \\
\text { de acuerdo }\end{array}$ \\
\hline & $\%$ & $\%$ & $\%$ & $\%$ \\
\hline $\begin{array}{l}\text { 1.- Siento que soy una persona } \\
\text { de valor }\end{array}$ & 51.3 & 34.0 & 9.4 & 5.2 \\
\hline $\begin{array}{l}\text { 2.- Definitivamente creo que soy } \\
\text { un fracaso }\end{array}$ & 5.2 & 8.9 & 29.3 & 56.5 \\
\hline $\begin{array}{l}\text { 3.- Siento que tengo muchas } \\
\text { buenas cualidades }\end{array}$ & 39.3 & 44.0 & 10.5 & 6.3 \\
\hline $\begin{array}{l}\text { 4.- Puedo hacer las cosas igual } \\
\text { que los demás }\end{array}$ & 40.3 & 34.0 & 16.2 & 9.4 \\
\hline $\begin{array}{l}\text { 5.- No tengo mucho porque sen- } \\
\text { tirme orgulloso }\end{array}$ & 15.2 & 31.9 & 20.4 & 32.5 \\
\hline $\begin{array}{l}\text { 6.- Tengo una actitud positiva } \\
\text { con mi persona }\end{array}$ & 50.3 & 35.1 & 8.4 & 6.3 \\
\hline $\begin{array}{l}\text { 7.-En todo, estoy satisfecha con- } \\
\text { migo }\end{array}$ & 44.5 & 39.3 & 9.9 & 6.3 \\
\hline $\begin{array}{l}\text { 8.- Desearía tener más respeto } \\
\text { conmigo misma }\end{array}$ & 42.4 & 37.2 & 11.0 & 9.4 \\
\hline $\begin{array}{l}\text { 9.- Algunas veces me siento in- } \\
\text { útil }\end{array}$ & 7.9 & 21.5 & 27.7 & 42.9 \\
\hline $\begin{array}{l}\text { 10.- Algunas veces, creo que no } \\
\text { sirvo para nada }\end{array}$ & 7.9 & 16.8 & 20.9 & 54.5 \\
\hline
\end{tabular}

Fuente: Elaboración propia 
Tabla 3. Nivel de autoestima y tipo de consumo por sexo

\begin{tabular}{cccc|cccc}
\hline \multirow{2}{*}{ Sexo } & \multicolumn{3}{c|}{ Nivel de Autoestima* } & \multicolumn{3}{c|}{ Tipo de Consumo de Alcohol ${ }^{* *}$} \\
\cline { 2 - 7 } & Baja & Media & Alta & Sensato & Dependiente & Dañino \\
\hline \multirow{2}{*}{ Masculino } & $\%$ & $\%$ & $\%$ & $\%$ & $\%$ & $\%$ \\
Femenino & 79.8 & 16.0 & 4.2 & 27.3 & 39.4 & 33.3 \\
\hline \multicolumn{2}{c}{ Total } & 81.4 & 11.3 & 7.3 & 38.8 & 32.7 & 28.5 \\
\hline Fuente: Elaboración propia & 80.8 & 13.6 & 5.5 & 34.1 & 35.4 & 30.5 \\
\hline
\end{tabular}

Tabla 4. Prevalencia global del consumo de alcohol por nivel de autoestima

\begin{tabular}{|c|c|c|c|c|c|c|c|c|c|}
\hline & & \multicolumn{8}{|c|}{ Nivel de Autoestima } \\
\hline \multicolumn{2}{|c|}{ Consumo de alcohol } & \multicolumn{2}{|c|}{ Baja } & \multicolumn{2}{|c|}{ Media } & \multicolumn{2}{|c|}{ Alta } & \multicolumn{2}{|c|}{ Total } \\
\hline & & $f$ & $\%$ & $f$ & $\%$ & $f$ & $\%$ & $f$ & $\%$ \\
\hline \multirow{2}{*}{ Alguna vez en la vida } & No & 52 & 27.2 & 11 & 5.8 & 3 & 1.6 & 66 & 34.6 \\
\hline & Si & 102 & 33.8 & 15 & 7.9 & 8 & 4.2 & 125 & 65.4 \\
\hline
\end{tabular}

Fuente: Elaboración propia

$\boldsymbol{n}=191$

\section{CONCLUSIÓN}

Los resultados permitieron corroborar que el consumo de alcohol en grupos vulnerables como los adolescentes va en incremento, constituyendo un creciente problema de salud que pudiera ocasionar daños biológicos, psicológicos y sociales, a corto mediano y largo plazo. Además de estar asociado a variables psicológicas como la autoestima, que juega un papel primordial en la adquisición o modificación de hábitos saludables o no, en este caso un factor protector ante el consumo de alcohol. La adolescencia es un período del ciclo de vida de los seres humanos donde se adquieren conductas, comportamientos y hábitos para la edad adulta. En esta etapa se conjugan una serie de factores que retardan o limitan dicha conducta; la autoestima, es una variable psicológica mediadora en la conducta del adolescente, por lo tanto, se debe de fortalecer con acciones específicas desde el ámbito familiar y social. Hay que considerar que los el desarrollo biológico, psicológico y social de los adolescentes pudiera verse afectado por la cantidad y frecuencias de la ingesta de bebidas alcohólicas, por lo tanto, en de vital importancia identificar las variables mediadoras, que permitan brindar las habilidades sociales necesarias para resistir a la presión de pares en relación al consumo de alcohol, tabaco y otras drogas.

\section{REFERENCIAS BIBLIOGRÁFICAS}

1. Organización Mundial de la Salud. Alcohol [Internet]. OMS; [Citado 17 Marzo 2017] Disponible en: http://www. who.int/mediacentre/factsheets/fs349/es/

2. Organización Mundial de la Salud. ¿Constituye el uso nocivo del alcohol un problema de salud pública? Preguntas y respuestas en línea [Internet]. OMS; [Citado 19 A b r i I 2017]. Disponible en: http://www.who.int/features/qa/66/es/ 3. Secretaria de Salud. Encuesta Nacional de Salud y Nutrición 2012. Resultados Nacionales. México: Secretaria de Salud; 2012. 196 p.

4. Acosta DL, Fernández AR, Pillon SC. Factores sociales para el uso de alcohol en adolescentes y jóvenes. Rev. Latino-Am. Enfermagem. 2011;19(Spe):771-81.

5. Rosenberg M. Society and the adolescent self-imagen. Princeton: Princeton University Press; c1965. 338 p.

6. Musitu G, Jiménez TI, Murgui S. Funcionamiento familiar, autoestima y consumo de sustancias en adolescentes: un modelo de mediación. Salud Pública México. 2007; 49:3-10.

7. Gámez Medina ME, Guzmán Facundo FR, Ahumada Cortez 
JG, Alonso Castillo MM, Gherardi Donato E. Autoestima y consumo de alcohol en adolescentes escolarizados. NURE Investigación. 2017;14(88):1-12.

8. Villarreal-González ME, Sánchez-Sosa JC, Musitu G, Varela R. El Consumo de Alcohol en Adolescentes Escolarizados: Propuesta de un Modelo Sociocomunitario. Psychosocial Intervention. 2010;19(3):253-64.

9. Armendáriz García NA, Rodríguez Aguilar L, Guzmán Facundo FR. Efecto de la autoestima sobre el consumo de tabaco y alcohol en adolescentes del área rural de Nuevo León, México. Revista Electrónica en Salud Mental, Alcohol y Drogas. 2008;4(1):1-12.

10. Burns N, Grove SK. The practice of nursing research. Appraisal, synthesis, and generation of evidence. 6th ed. St. Louis: Elsevier Saunders; c2009. 729 p.

11.Burns N, Grove SK. Investigación en Enfermería. 3ra ed. St. Louis: Elsevier Saunders; c2004. 578 p.

12. Elashoff DJ, Dixon JW, Crede MK, Fothenringham N. nQuery Advisor (version 4.0) Copyrigth [Software estadístico para PC]. Los Angeles: Statistical Solutions; 2000.

13. Ley General de Salud en Materia de Investigación para la Salud. Diario Oficial de la Federación, (1987).

14. World Health Organization. The alcohol use disorders identification test. Geneva: WHO; 2001. 40 p.

15. De la Fuente JR, Kershenobich D, Narro J, Tapia Conyer R, Meneses González F, Gutiérrez H, et al. El alcoholismo como problema médico. Revista Facultad de Medicina. UNAM. 1992;35(2):47-74.

16. López-Cisneros MA, Villar Luis MA, Alonso Castillo MM, Alonso Castillo MTJ, Rodríguez Aguilar L. Actitud ante el consumo y no consumo de alcohol en estudiantes de preparatoria-México. Rev Esc Enferm USP. 2013;47(4): 81521.

17. Telumbre-Terrero JY, Sánchez-Jaimes BE. Consumo de alcohol en adolescentes del estado de Guerrero, México. Health and Adicctions. 2015;15(1):79-86.

18. Rosenberg M. Society and the adolescent self-imagen. Princeton: Princeton University Press; c1965. 338 p.

19. Álvarez-Aguirre A, Alonso-Castillo MM, Guidorizzi-Zanetti AC. Consumo de alcohol y autoestima en adolescentes. Rev. Latino-Am. Enfermagem. 2010;18(Spec);634-40.

20. Morales F, Cabrera M, Pérez Barajas CJ, Amaro ML. El consumo de alcohol en adolescentes de una secundaria de Ciudad Nezahualcóyotl Frecuencia y características sociales, 2015. VERTIENTES Revista Especializada en Ciencias de la Salud. 2015;18(1):8-15.

21. Bustos M, Villatoro JA, Oliva N, López MA, Fregoso DA, Medina-Mora ME. Consumo de tabaco en adolescentes y jóvenes de México que no estudian y no trabajan. Revista Internacional de Investigación en Adicciones. 2015;1(1):3340.

22. Blázquez-Morales MS, Pavón-León P, GogeascoecheaTrejo MC, P Beverido Sustaeta P. Consumo de alcohol y tabaco en adolescentes de secundaria del estado de Veracruz. Rev. Med. UV. 2012;12(1):25-31.

23. Villegas-Pantoja MA, Alonso-Castillo MM, Benavides-
Torres RA, Guzmán-Facundo FR. Consumo de alcohol y funciones ejecutivas en adolescentes: una revisión sistemática. Aquichan. 2013;13(2):234-46.

24. Instituto Nacional sobre el Abuso de Drogas. Las drogas, el cerebro y el comportamiento: La ciencia de la adicción [Internet]. NIH; 2008 [Citado 20 Marzo 2018]. 36 p. Disponible en: https://www.drugabuse.gov/sites/default/ files/soa_spanish.pdf

25. Zacarés JJ, Iborra A, Tomás JM, Serra E. El desarrollo de la identidad en la adolescencia y adultez emergente: Una comparación de la identidad global frente a la identidad en dominios específicos. Anales de psicología. 2009;25(2):31629.

26. Fondo de las Naciones Unidas para la Infancia. Adolescencia una etapa fundamental [Internet]. New York: Fondo de las Naciones Unidas para la Infancia (UNICEF); 2002 [Citado 20 Enero 2018]. 44 p. Disponible en: https:// www.unicef.org/ecuador/pub_adolescence_sp.pdf

27. Rodríguez C, Caño A. Autoestima en la adolescencia: análisis y estrategias de intervención. International Journal of Psychology and Psychological Therapy. 2012;12(3):389-403.

28. Ferrel FR, Vélez J, Ferrel LF. Factores psicológicos en adolescentes escolarizados con bajo rendimiento académico: depresión y autoestima. Revista Encuentros, Universidad Autónoma del Caribe. 2014;12(2):35-47.

29. Milicic N, Gorostegui ME. Género y autoestima: un análisis de las diferencias por sexo en una muestra de estudiantes de educación general básica. Psykhe. 2011;2(1):1-10.

30. Padilla MT, García S, Suárez M. Diferencias de género en el autoconcepto general y académico de estudiantes de $4^{\circ}$ de ESO. Revista de Educación. 2010;352:495-515.

31. Domínguez C, Telumbre-Terrero J, Sánchez B, López M, Carranza A. Características del consumo de alcohol en mujeres adolescentes de Chilpancingo, Guerrero, México. Ciencia y Humanismo en la Salud. 2016;3(1):4-13.

32. Gutiérrez M, Romero I. Resiliencia, bienestar subjetivo y actitudes de los adolescentes hacia el consumo de drogas en Angola. Anales de Psicología. 2014;30(2):608-19. 